\title{
Adhesive and hydrophobic properties of the selected LAB isolated from gastrointestinal tract of farming animals*
}

\author{
Katarzyna Grajek ${ }^{\bowtie}$, Anna Sip², Joanna Foksowicz-Flaczyk¹, Anna Dobrowolska² and \\ Agnieszka Wita²
}

'Department of Innovative Biomaterials and Nanotechnologies, Institute of Natural Fibres and Medicinal Plants, Poznań, Poland; 2 Department of Biotechnology and Food Microbiology, University of Life Sciences, Poland, Poznań, Poland

The aim of this study was to determine the degree of adhesion and hydrophobicity of some strains of lactic acid bacteria (LAB) with proven antagonistic properties against pathogenic bacteria. Studies were performed using-LAB strains isolated from the gastrointestinal tract of calves and piglets. These strains exhibited an antibacterial activity against pathogenic strains of Clostridium perfringens and Escherichia coli. Cell adhesiveness was examined in relation to the porcine and bovine mucin. Our analyses had shown that the tested microorganisms demonstrated a degree of adhesion in the range of $32.00-40.00 \%$ for strains isolated from calves, and $34.00-40.00 \%$ for strains isolated from pigs. The hydrophobicity of tested bacteria was in the range of $31.00 \%-$ $44.00 \%$ for strains isolated from pigs, and $26.00 \%-$ $42.00 \%$ for strains obtained from calves. The best mucin adhesion ability was found for bacterial strains belonging to the Enterococcus genus isolated from calves. Taking into account porcine isolates, the best adhesion was observed for the Leuconostoc bacterial strains. Among tested strains, the highest hydrophobicity, measured in relation to hexadecane, was recorded for the bacterial strains belonging to the Leuconostoc sp. isolated from piglets and a of Lactobacillus sp. bacterial strain isolated from calves.

Key words: lactic acid bacteria, adhesion, hydrophobicity

Received: 24 July, 2015; revised: 07 March, 2016; accepted: 06 May, 2016; available on-line: 25 May, 2016

\section{INTRODUCTION}

Probiotics are food products containing living cells of microorganisms that provide beneficial effects to the health of their consumer (Ouwehand, 2001). The LAB strains, the main component of probiotic preparations, produce a series of anti-microbial compounds such as organic acids (acetic acid, lactic acid), bacteriocins, hydrogen peroxide, carbon dioxide and others. Whenever a microorganism is to be recognized as a probiotic, it must meet stringent selection criteria. These criteria include: resistance to acids and bile salts, survival during passage through the gastrointestinal tract, ability to colonize the intestine, antagonistic properties against pathogenic microorganisms, stimulation of the immune system, capability to degrade toxic substances and improve absorption of certain nutrients, and they have to be accompanied by good technological properties (Ouwehand, 1999; Ouwehand, 2001). Adhesion of a microorganism to the surface of the intestinal mucus and epithelial cells is one of the most import- ant probiotic characteristics. It influences the antimicrobial and immunomodulating effects that depend on temporary colonization of the digestive tract by probiotic bacteria (Rinkinen et al., 2003). Physical and chemical properties of the surface of bacterial cell depend mainly on its hydrophobicity (Pan et al., 2006). Microorganisms have developed on their cell surfaces a range of hydrophobic components referred to as adhesins, such as proteins - or, less commonly, polysaccharides, teichoic and fatty acids that enable them to bind covalently to the surface of the intestinal epithelium and mucin. The hydrophobic residues contribute to the adhesive reaction between the microorganisms and a suitable substrate, e.g. bacterial adhesins recognize the carbohydrate residues (mannose, galactose and fructose) of glycoproteins and glycolipids on the epithelial surface (Doyle, 2000). The aim of this study was to determine the adhesion properties and hydrophobicity of selected strains that show antagonistic properties against pathogenic strains of Clostridium perfringens and Escherichia coli. The study was conducted on strains isolated from the skin and mouth cavities of pigs and calves. Adhesiveness of the tested strains was measured in relation to commercially available porcine and bovine mucin. A working hypothesis that adhesion properties are positively correlated with hydrophobicity of bacterial cells was verified.

\section{MATERIALS AND METHODS}

Microorganisms. Research on adhesion properties and hydrophobicity was carried out using LAB strains from the collection of the Department of Biotechnology and Food Microbiology, the Poznan University of Life Sciences. These strains were isolated from animals and they exhibited an antibacterial activity against pathogenic strains of Clostridium perfringens and Escherichia coli. The strains isolated from pigs mainly belonged to the Enterococcus sp. genus, whereas those from calves were Lactobacillus spp.

Chemicals and reagents. In the study the following reagents were used: PBS and HEPES buffers (Sigma-Alderich, Germany), MRS medium (OXOID), N- hexadecane (Sigma-Alderich, Germany), mucin from calves and pigs (Sigma-Alderich, Germany). Mucins were diluted in the HEPES buffer to obtain a final concentration of 0.5 $\mathrm{mg} / \mathrm{ml}$.

e-mail: katarzyna.grajek@gmail.com

*The results were presented at the 6th International Weigl Conference on Microbiology, Gdańsk, Poland (8-10 July, 2015).

Abbreviations: PBS, phosphate-buffered saline; HEPES,

4-(2-hydroxyethyl)-1-piperazineethanesulfonic acid 
Bacteria preparation. Strains of the examined LAB groups were cultured on liquid MRS media and incubated at $37^{\circ} \mathrm{C}$ for $24 \mathrm{~h}$. Lactic acid bacteria cultures were grown to a density of $0.5^{\circ} \mathrm{McF}\left(10^{6}-10^{8} \mathrm{cfu} / \mathrm{ml}\right)$, as determined with a DEN-1B densitometer (0.00-15.00 McFarland units by BIOSAN).

Adhesiveness testing. The adhesive capabilities of the tested lactic acid bacteria to porcine and bovine mucins were assessed on a 96-well plate. For this purpose, a relevant amount of mucin was weighed and dissolved in the HEPES buffer under sterile conditions to a final mucin concentration of $0.05 \mathrm{mg} / \mathrm{ml}$. The bottom of each well was covered with porcine or bovine mucin and incubated overnight at $4^{\circ} \mathrm{C}$. After this incubation period, the mucin solution was removed and wells were washed 2-3 times with HEPES to remove unbound mucin. Bovine mucin which remained attached to the bottom of the wells was used for bovine strain analyses, while swine mucin was used for porcine strains.

In order to determine the degree of adhesion, quantitative plate cultures were performed. Standardized bacterial suspensions $\left(10^{6}-10^{9} \mathrm{CFU} / \mathrm{ml}\right.$, which were prepared in $2 \mathrm{ml}$ of liquid PBS buffer) were transferred into wells covered with bovine mucin and incubated at $37^{\circ} \mathrm{C}$ for 1 h. Next, dilutions were prepared $\left(10^{-4}\right.$ to $10^{-8}$ of $0.5^{\circ}$ $\mathrm{McF}$ bacterial suspension), transferred into Petri dishes, and incubated on solid MRS $\left(2 \%\right.$ agar) at $37^{\circ} \mathrm{C}$ for $72 \mathrm{~h}$. Reference plate cultures that were not treated with mucin were carried out in-parallel. The degree of adhesion was determined by the difference between the counts of bacteria remaining after mucin treatment and the reference cultures. The results are shown as a percentage of bacteria attached to the surface of the intestinal mucus.

Hydrophobicity determination. Cultures of bacteria were centrifuged (Biofuge primo $\mathrm{R}$ centrifuge Heraeus) at $4000 \mathrm{rpm}$ for 10 minutes. After centrifugation, the supernatant was removed and the cell pellet was resuspended in PBS ( $\mathrm{pH}$ 7.4). This procedure was repeated one more time. Then, the cell suspension was diluted with the PBS buffer to an absorbance value of 1.0, when measured at $560 \mathrm{~nm}$. Next, $600 \mu \mathrm{l}$ of n-hexadecane was added to each sample, mixed (vortex-type mixer) for $2 \mathrm{~min}$ and incubated at $37^{\circ} \mathrm{C}$ until phase separation was observed (15 to $60 \mathrm{~min}$ ). Afterwards, the optical density of bacterial cells in the collected aqueous phases was determined spectrophotometrically at the $560 \mathrm{~nm}$ wavelength.

The percentage of hydrophobicity was calculated from the formula:

$\mathrm{H} \%=\left(\mathrm{A}_{0}-\mathrm{A}\right) * 100 \% / \mathrm{A}_{0}$

where:

$A_{0}$ - is the absorbance of lactic acid bacteria cultures prior to the addition of $n$-hexadecane

A - is the absorbance after the addition of n-hexadecane measured at the aqueous phase

Statistical calculations. In order to determine the correlation between adhesion properties and cell hydrophobicity, statistical analysis was performed. Separate analyses were performed for strains isolated from pigs and calves.

\section{RESULTS AND DISCUSSION}

\section{Adhesion}

The ability of bacteria to attach to the mucin matrix was studied, which facilitates their adhesion to intestinal

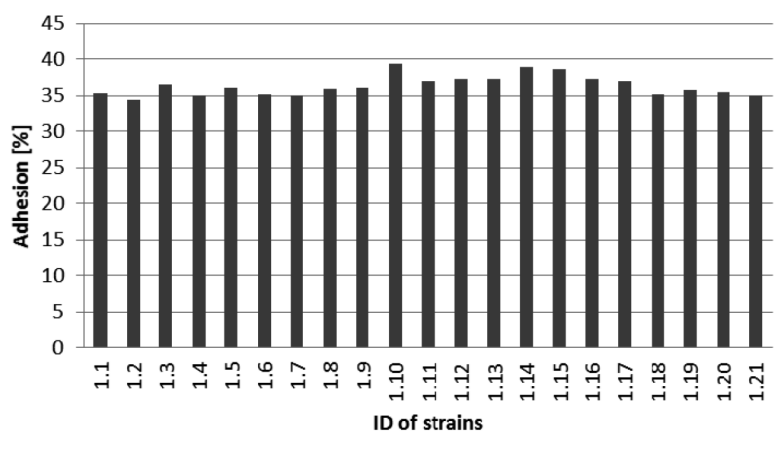

Figure 1. Adhesion to porcine mucin of bacteria isolated from pigs.

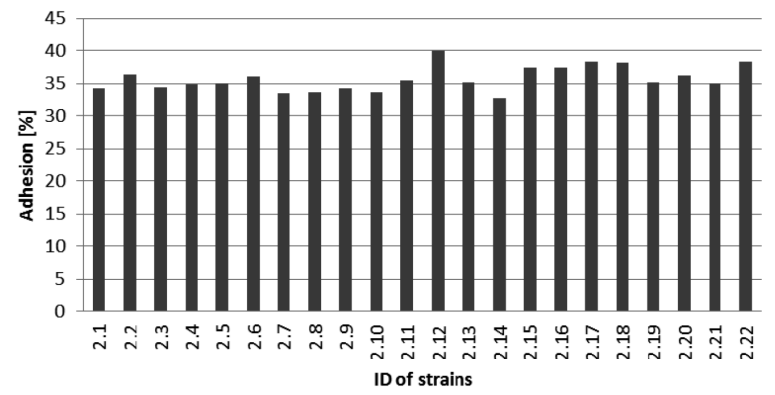

Figure 2. Adhesion ability of bacterial strains isolated from calves to bovine mucin.

epithelium. The test consisted of a temporary exposure of bacterial cells to the surface of mucin layers prepared in 96-well plates. The adhesion value was expressed as the percentage of cells attached to mucin in relation to the number of cells introduced into the wells.

\section{Adhesion of bacteria isolated from pigs}

The tested LAB strains isolated from pigs showed small differences (about 5\%) between the highest and lowest value of adhesion. The highest adhesion ability, amounting to $39.37 \%$, was observed for the Leuconostoc sp. 1.10 strain. The lowest adhesion of $34.37 \%$ was recorded for Enterococcus sp. 1.2 strain. Very small differences in mucin adhesion were observed among the tested strains of Enterococcus spp. and Leuconostoc spp. Differences between the lowest and the highest value of adhesion among Enterococcus spp. strains ranged from $0.71 \%$ to $1.66 \%$. The difference between the extreme values for Leuconostoc spp. reached $2.51 \%$. The results of the adhesion ability of LAB strains isolated from pigs are shown on Fig. 1.s

\section{Adhesion of bacteria isolated from calves}

The tested strains of microorganisms isolated from calves showed a greater difference between the lowest and highest values of adhesion than the strains obtained from pigs. The highest value of adhesion was recorded for the Enterococcus sp. 2.12 strain (39.91\%), while it was lowest for the Lactobacillus sp. 2.14 strain $(32.72 \%)$. The analysis of adhesion properties of strains belonging to the Enterococcus spp. and Lactobacillus spp. indicated that among the species of the strains obtained from calves, the disparity between results was more pronounced than in the case of strains obtained from pigs. 


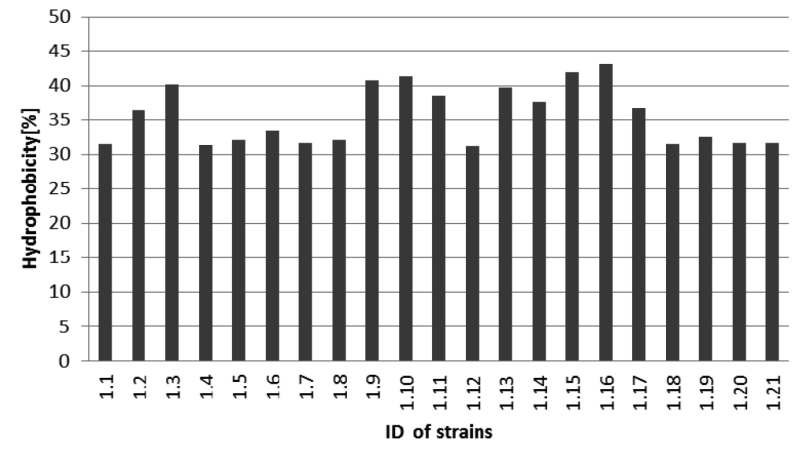

Figure 3. Hydrophobicity of the bacteria isolated from pigs.

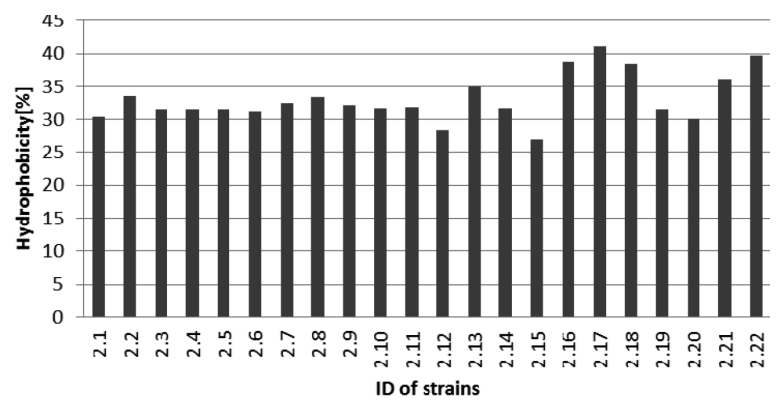

Figure 4. Hydrophobicity of the bacterial cells isolated from calves.

The results of adhesion properties of bacteria isolated from calves to the bovine mucin are presented in Fig. 2.

\section{Hydrophobicity}

This analysis was conducted using the MATH method (microbial adhesion to hydrocarbon) based on the transfer of microbial cells into the hexadecane phase. The results were expressed as a percentage of the cell population which had passed into the hydrophobic phase of the solvent.

\section{Hydrophobicity of bacteria isolated from pigs}

When analyzing the results, it can be concluded that strains 1.2-1.9 and 1.18-1.21 belonging to the Enterococcus spp., showed a noticeable difference between the highest and lowest value of hydrophobicity. The values of this parameter ranged from $40.80 \%$ (strain 1.9) to $31.36 \%$ (strain 1.4). The difference was 9.44 percentage points. Similar results were obtained for the Leuconostoc strains 1.10-1.17, which demonstrated variation of hydrophobicity at the level of 10.82 percentage points (Fig. 3).

\section{Hydrophobicity of bacteria isolated from calves}

The tested strains isolated from calves had shown a much greater variation in hydrophobicity than the strains isolated from pigs. Differences in cell hydrophobicity of different strains obtained from calves were as high as $14.08 \%$. Relatively low hydrophobicity was demonstrated by the Enterococcus spp. 2.1-2.13 strains, in which case values ranged from $34.9 \%$ (strain 2.13 ) to $28.43 \%$ (strain 2.12). The difference was 6.47 percentage points. For strains belonging to the Lactobacillus spp. (2.14-2.19) the difference between the highest hydrophobicity value $(41.07 \%)$ obtained for strain 2.17 and the lowest
(26.99\%) exhibited by strain 2.15 , reached 14.08 percentage points (Fig. 4).

\section{A correlation between the adhesion and hydrophobicity of tested strains}

Statistical calculations indicated that in the case of bacteria isolated from pigs, the correlation between hydrophobicity of the tested strains and their adhesion to porcine mucin was very small. The correlation coefficient was $\mathrm{p}=0.00273$ at $\mathrm{r}=0.61$ (Fig. 5).

In the case of bacteria isolated from calves, there was no correlation between hydrophobicity and adhesion of the tested strains. The correlation coefficient was $p=0.184471$ at $r=0.301265$. Hydrophobicity and adhesion of the tested strains obtained from calves are shown on Fig. 6.

According to literature sources, MATH (microbial adherence to hydrocarbon) is the most commonly used method. It is based on the determination of hydrophobicity of a bacterial surface to hexadecane, which serves as the hydrocarbon model (Oliveira et al., 2001). Hydrophobicity affects physical and chemical properties of bacterial cells. Pan and coworkers (2006) also studied bacterial hydrophobicity using the MATH method with hexadecane. They tested 43 strains of bacteria isolated from pigs (21 strains) and calves (22 strains). The bacteria were characterized by varying hydrophobicity, which ranged from $26.99 \%$ to $43.13 \%$. In bacteria isolated from pigs this value was higher than in bacteria from calves. Literature data also show large variability in hydrophobicity values obtained for different tested microorganisms. Pan and coworkers (2006) examined hydrophobicity of 23 Bifidobacterium strains and observed low hydrophobicity values. Only $13.04 \%$ of the strains reached hydrophobicity above $20 \%$. The highest value

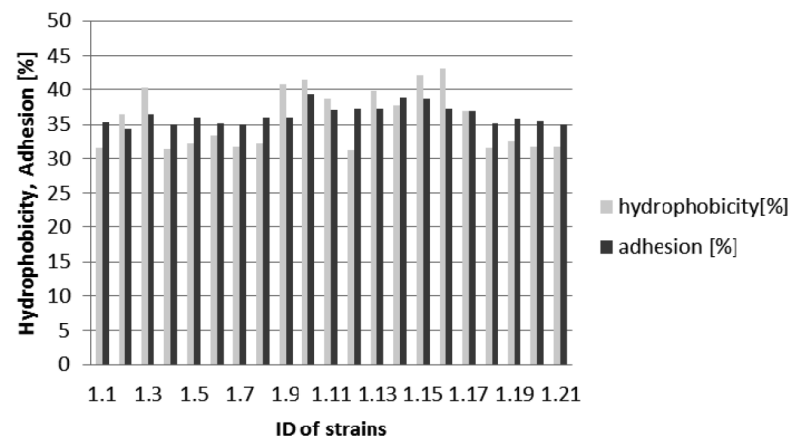

Figure 5. Hydrophobicity of the tested strains isolated from pigs compared to their adhesion to porcine mucin.

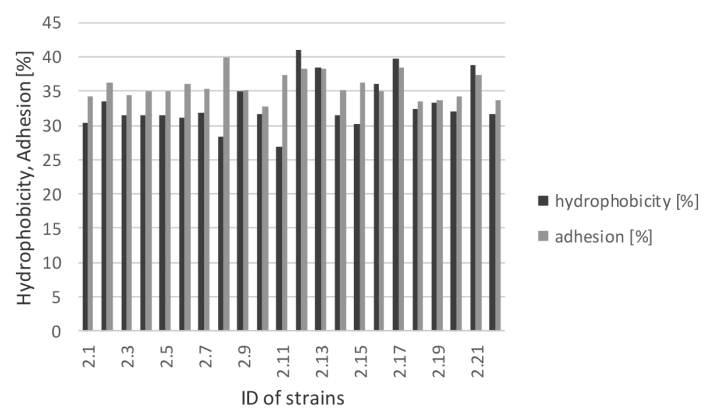

Figure 6. Hydrophobicity of the tested strains isolated from calves compared to their adhesion to the bovine mucin. 
recorded was 37.2\%. Giaouris and coworkers (2009) examined 50 strains of $L$. lactis. The authors reported very high values of hydrophobicity, reaching 48-88\%. Strains showing hydrophobicity values below $40 \%$ were hydrophilic.

Adhesion to mucin is one of the most important features of probiotic bacteria and it depends on the bacterial cell composition and structure. Positive effects on the host, such as antagonism to pathogenic microorganisms or the regulation of the immune system, are associated with colonization of the intestinal mucus by probiotic bacteria (Ouwehand et al., 1999a; Ouwehand et al., 1999b; Pan et al., 2006; Rinkinen et al., 2003). In vitro study of bacterial adhesion to mucin is a proven method of evaluating adhesion capacity of probiotic bacteria (Kirjavainen et al., 1998; Rinkinen et al., 2003).

Adhesion assays commonly utilize adhesion of bacteria to the surface of intestinal epithelial cells or intestinal mucus produced by these cells and covering them with a thin layer (Ouwehand et al., 1999). In the studies conducted here, adhesion to mucus was determined for 43 LAB strains, of which 21 were obtained from pigs and 22 strains from calves. Overall, adhesion values obtained for the tested strains ranged between 32.72 and 39.91\% of attached bacteria. In the case of strains obtained from pigs, it ranged between 34.37-39.37\%, while for bacteria isolated from calves it ranged between 32.72-39.91\%, respectively. In this study, adhesion values recorded for analyzed bacterial strains are comparable with literature data. In the study by Ouwehand and coworkers (1999), adhesion to human mucin by LAB isolated from individuals of varying age ranged from $2.5 \%$ to $42.5 \%$. In other studies conducted by these authors, adhesion abilities of tested bacterial strains reached a maximum of $19.3 \%$. Rinkinen (2003) reported adhesion values in the range of $33.4-42.1 \%$ for L. rhamnosus and $21 \%$ for E. faecium in a paper concerning LAB adhesion to mucin from various sources. Ouwehand and coworkers (2001) studied adhesion of lactic acid bacteria (Lactobacillus brevis PELI, L. reuteri ING1, L. rhamnosus VTT E-800 and L. rhamnosus LC-705) to a glycoprotein obtained from the ileum and glycoproteins isolated from human feces. The study showed that three strains exhibited adhesion capability ranging from $34 \%$ to $36.5 \%$ to glycoproteins of the ileum, while in the case of glycoproteins isolated from stool the values were $31.1-37 \%$. The two other strains showed adhesion values of $19.3 \%$ and $0.79 \%$ to intestinal epithelial cells in an in vitro experiment.

Hydrophobic properties of bacterial cells are considered to be an important factor increasing their adhesion to the intestinal surface. They depend on the composition and structure of the bacterial cell walls, especially on the presence of hydrophobic proteins (Pan et al., 2006). The study presented here indicates a correlation between bacterial adhesion to mucin and cell hydrophobicity in strains isolated from pigs (correlation coefficient $r=0.61$; $\mathrm{p}=0.00273)$.

This corresponds to the results of Pan and coworkers (2006), who found that adhesion (measured using Caco2) and hydrophobicity of the tested bacteria of the Bifidobacterium genus are correlated to a certain degree. High adhesion values were accompanied by a relatively high hydrophobicity of approx $47 \%$. The researchers reported the correlation coefficient at $r^{2}=0.78$. In our study, statistical analysis indicated no correlation between mucin adhesion parameters and hydrophobicity of the tested strains isolated from calves. This in turn corresponds with the results of Ouwehand and coworkers (1999), who observed no correlation between hydrophobicity of the cell surface of tested probiotic bacteria cells and their adhesion properties determined using the four types of mucin.

\section{CONCLUSIONS}

In summary, these experiments revealed that there is a high positive correlation between adhesion properties of lactic acid bacteria tested and their cell surface hydrophobicity. All isolated strains of LAB with antagonistic activity against pathogenic strains of Escherichia coli and Clostridium perfringens had shown an ability to adhere to porcine and bovine mucin. The tested microorganisms demonstrated a degree of adhesion in the range of $32-40 \%$ of cells. The hydrophobicity of the tested bacteria ranged from $26 \%$ to $42 \%$ of cells, depending on the origin of the isolated strains.

\section{Acknowledgements}

This work was supported by the 1st Call of Applied Research Programme of the National Centre for Research and Development, Project "The development of eubiotic preparations for livestock" (Acronym: EUBIOTYKI, PBS1/A8/10/2012).

\section{REFERENCES}

Doyle RJ (2000) Contribution of the hydrophobic effect to microbial infection. Microbes and Infection 2: 391-400. DOI: 10.1016/S12864579(00)00328-2.

Giaouris E, Chapot-Chartier MP, Briandet R (2009) Surface physicochemical analysis of natural Lactococcus lactis strains reveals the existence of hydrophobic and low charged strains with altered adhesive properties. Int J Food Microbiol 131: 2-9. DOI: 10.1016/j.ijfoodmicro.2008.09.006.

Kirjavainen PV, Ouwehand AC, Isolauri E, Salminen SJ (1998) The ability of probiotic bacteria to bind to human intestinal mucus. FEMS Microbiol Lett 167: 185-189. DOI: 10.1111/j.15746968.1998.tb13226.x 185-189.

Oliveira R, Azeredo J, Texeira P, Fonseca AP (2001) The role of hydrophobicity in bacterial adhesion. Bioline 11-22.

Ouwehand AC, Kirjavainen PV, Shortt C, Salminen S (1999) Probiotics: mechanisms and established effects. Int Dairy J 9: 43-52.

Ouwehand AC, Kirjavainen PV, Grönlund MM, Isolauri E, Salminen SJ (1999a) Adhesion of probiotic micro-organisms to intestinal mucus. Int Dairy J 9: 623-630. DOI: 10.1046/j.1472-765x.2000.00773.x.

Ouwehand AC, Isolauri E, Kirjavainen PV, Salminen SJ (1999b) Adhesion of four Bifidobacterium strains to human intestinal mucus for subjects in different age groups. FEMS Microbiol Lett 172: 61-64. DOI: $10.1111 /$ j.1574-6968.1999.tb13450.x.

Ouwehand AC, Tuomola EM, Tölkko S, Salminen S (2001) Assessment of adhesion properties of novel probiotic strains to human intestinal mucus. Int J Food Microbiol 64: 119-126. DOI: 10.1016/ S0168-1605(00)00440-2.

Pan WH, Li PL, Liu Z (2006) The correlation between surface hydrophobicity and adherence o Bifidobacterium strains from centenarians faeces. Anaerobe 12: 148-152.

Rinkinen M, Westermarck E, Salminen S, Ouwehand AC (2003) Absence of host specificity for in vitro adhesion of probiotic lactic acid bacteria to intestinal mucus. Vet Microbiol 97: 55-61. DOI:10.1016/ S0378-1135(03)00183-4. 